\title{
Un Estudio Teórico de la Estructura Electrónica y las Propiedades Dieléctricas de $\mathrm{B}-\mathrm{Nb}_{2} \mathrm{O}_{5}$
}

\author{
Camilo Valencia-Balvín ${ }^{1}$ \\ Santiago Pérez-Walton ${ }^{2}$ \\ Jorge Osorio-Guillén ${ }^{3}$
}

\section{Resumen}

En este trabajo hemos investigado teóricamente las propiedades electrónicas y ópticas del óxido de niobio $\mathrm{Nb}_{2} \mathrm{O}_{5}$ para una de sus fases cristalinas, a saber la fase B. Usamos la Teoría de los Funcionales de la Densidad junto con la Aproximación del Gradiente Generalizado con uno de sus nuevos funcionales diseñado para sólidos: PBEsol. La banda prohibida calculada es indirecta $\left(E_{g}=2,54 \mathrm{eV}\right)$, con el máximo de la banda de valencia localizado en $\Gamma$ y el mínimo de la banda de conducción en $(\overline{1} / 3,1 / 3,1 / 3)$. También calculamos la parte real e imaginaria del tensor dieléctrico, el índice de refracción, la reflectividad, la transmitancia y la parte real de la conductividad óptica. El valor calculado del índice de refracción es 2,52 , que está en buena correspondencia con el valor experimental de 2,64 .

\section{Palabras clave}

Propiedades electrónicas, propiedades ópticas, óxido de niobio, DFT, PBEsol.

1 Centro de Investigación, Instituto Tecnológico Metropolitano, Medellín-

Colombia, camilovalencia@itm.edu.co

2 Centro de Investigación, Instituto Tecnológico Metropolitano, Medellín-

Colombia, santiagoperez@itm.edu.co

3 Instituto de Física, Universidad de Antioquia, Medellín-Colombia, jorge.osorio@fisica.udea.edu.co

Fecha de recepción: 28 de febrero de 2011

Fecha de aceptación: 29 de agosto de 2011 
[104] Un Estudio Teórico de la Estructura Electrónica y las Propiedlades Dieléctricas de $\mathrm{B}-\mathrm{Nb}_{2} \mathrm{O}_{3}$

\section{Abstract}

In this work we have investigated theoretically the electronic and optical properties of niobium-oxide $\mathrm{Nb}_{2} \mathrm{O}_{5}$ for one of its crystalline phases, namely the B-phase. We have used Density Functional Theory along with the Generalized Gradient Approximation with one of its new modified functional targeted for solids: PBEsol. The calculated band gap is indirect $\left(E_{g}=2,54 \mathrm{eV}\right)$, with the valence band maximum located at $\Gamma$ and the conduction band minimum located at $(\overline{1} / 3,1 / 3,1 / 3)$. We present also the calculated real and imaginary part of the dielectric tensor, the index of refraction, the reflectivity, the transmittance and the real part of the optical conductivity. The calculated value of the index of refraction is 2,52, which is in good agreement with the experimental value of 2,64 .

\section{Keywords}

Electronic properties, optical properties, niobium oxide, DFT, PBEsol. 


\section{INTRODUCCIÓN}

$\mathrm{El} \mathrm{Nb}_{2} \mathrm{O}_{5}$ es uno de los compuestos con mayor capacidad dieléctrica para ser utilizado en varias aplicaciones electrónicas tales como transistores de puertas dieléctricas y capacitores de memoria con acceso dinámico aleatorio (DRAM) (Clima et al., 2010). Por otra parte, las propiedades foto-catalíticas de este material han sido estudiadas por varios autores (Sayama et al., 1998; Prado et al., 2008; Santana \& Machado, 2008). Estas propiedades fotocatalíticas se deben a la creación por irradiación de un par electrón-hueco en la superficie; como consecuencia de la transferencia de carga entre el par electrón-hueco, se favorece la absorción de moléculas en la superficie del semiconductor. Este hecho puede ser utilizado en la foto-degradación de contaminantes en la superficie de $\mathrm{Nb}_{2} \mathrm{O}_{5}$ (Esteves et al., 2008). Además, el $\mathrm{Nb}_{2} \mathrm{O}_{5}$ es también utilizado como un catalizador ácido tolerante al agua, el cual es usado en diversas actividades acuáticas que involucran reacciones como la hidrólisis, la deshidratación y la hidratación (Tanabe \& Okasaki, 1995; Tanabe, 2003). Es importante resaltar que el cálculo de los parámetros cristalográficos, las propiedades electrónicas, dieléctricas y ópticas de este compuesto por medio de simulaciones atomísticas es una herramienta importante para la comprensión de sus propiedades físicas y su utilización en estas aplicaciones.

$\mathrm{El} \mathrm{Nb}_{2} \mathrm{O}_{5}$ existe en varias fases cristalinas estables dependiendo de la presión y la temperatura a la que esté sometido. Algunas de estas fases cristalinas son las siguientes: fase B (Laves et al., 1964; Wadsley \& Anderson, 1970), que cristaliza en una estructura monoclínica, la cual es estable en el rango de presiones entre 5 y $6 \mathrm{GPa}$ y de temperaturas entre 900 y $1000^{\circ} \mathrm{C}$; fase Z (Zibrov et al., 1998), que también es monoclínica y coexiste con la fase $\mathrm{B}$ a una presión de 7,5 GPa y en un rango de temperaturas entre $800 \mathrm{y}$ $1100^{\circ} \mathrm{C}$ y la fase $\mathrm{T}$ (Kato \& Tamura, 1975), estable en un rango de presiones entre 7,5 y $8 \mathrm{GPa}$ y de temperaturas entre $1200 \mathrm{y}$ $1300^{\circ} \mathrm{C}$, y cristaliza en una estructura ortorrómbica.

$\mathrm{El}$ artículo está organizado de la siguiente forma: en la sección 2 se describe la metodología utilizada para estudiar este material, en la sección 3 se presenta los resultados y se discute las propiedades cristalográficas, electrónicas, dieléctricas y ópticas, y final- 
mente en la sección 4, se presenta las conclusiones de esta investigación.

\section{MÉTODO COMPUTACIONAL}

Para calcular las propiedades de este compuesto, se comienza calculando la energía total como una función del volumen de la celda unitaria y se relaja para cada volumen sus posiciones iónicas y la forma de la celda unitaria (i.e., $b / a, c / a, \mathrm{y} \beta$ ). Luego, estas energías fueron ajustadas por medio de la ecuación de estado (EOS) de Birch--Murnaghan para sólidos (Murnaghan, 1944; Birch, 1947). A partir de la EOS, se obtuvo el módulo volumétrico $B$, su primera derivada $B^{\prime}$ y el volumen de equilibrio $\Omega_{0}$. A continuación, para $\Omega_{0}$ se realiza el cálculo de las propiedades electrónicas y del tensor dieléctrico. Todas las energías totales, las fuerzas inter-iónicas y el tensor de esfuerzo han sido computadas por medio de cálculos de primeros-principios a partir la Teoría de los Funcionales de la Densidad (DFT), usando el método del proyector de ondas aumentadas (PAW) (Blöchl, 1994) y la aproximación del Gradiente Generalizado con una de sus nuevas parametrizaciones diseñada especialmente para sólidos GGA-PBEsol (Perdew et al., 2008), tal como está implementado en el código de VASP (Kresse \& Joubert, 1999).

Se utilizó como configuración atómica de referencia en la construcción de los potenciales PAW $4 p^{6} 4 d^{4} 5 s^{1}$ para el $\mathrm{Nb}$ y $2 s^{2} 2 p^{4}$ para el $\mathrm{O}$, donde solo los electrones que aparecen explícitamente en la configuración electrónica son tratados como electrones de valencia. La energía de corte en la expansión de las ondas planas fue de 520 $\mathrm{eV}$, donde la energía total se convergió a $1 \mathrm{meV} / c e l d a$ unitaria. En la relajación de los iones y la forma de la celda unitaria, se realizó la integración en la primera zona de Brillouin con una malla del tipo Monkhorst 2x2x2 y usando una Gaussiana de ancho 0,01 eV (la fuerza sobre los iones fue convergida a $1 \mathrm{meV} / \AA$ ). Para el cálculo de las energías totales, la densidad de carga y las propiedades electrónicas y ópticas, se usó una malla $6 \times 6 \times 6$ centrada en $\Gamma$, se utilizó el método del tetraedro e incluyendo las correcciones de Blöchl. Una vez el estado base electrónico fue determinado, se 
calculó la parte imaginaria del tensor dieléctrico por medio del formalismo PAW, donde se desprecian las componentes fuera de la diagonal (efectos de campo local) y se usó la siguiente expresión (1) (Gajdos et al., 2006):

$$
\begin{aligned}
\varepsilon_{\alpha \beta}^{(2)}(\omega)= & \frac{4 \pi^{2} e^{2}}{\Omega_{0}} \lim _{q \rightarrow 0} \frac{1}{q^{2}} \\
& \sum_{c, v, \boldsymbol{k}} 2 \omega_{\boldsymbol{k}} \delta\left(\epsilon_{c \boldsymbol{k}}-\epsilon_{v \boldsymbol{k}}-\omega\right)\left\langle u_{c \boldsymbol{k}+e_{\alpha} \boldsymbol{q}} \mid u_{v \boldsymbol{k}}\right\rangle\left\langle u_{c \boldsymbol{k}+e_{\beta} \boldsymbol{q}} \mid u_{v \boldsymbol{k}}\right\rangle^{*}
\end{aligned}
$$

donde en (1) los índices $c$ y $v$ corresponden a los estados de las bandas de conducción y valencia, respectivamente, $e$ es la carga del electrón, $\Omega_{0}$ es el volumen del cristal $\epsilon$, son los autovalores de Khon-Sham, $u$ son ondas planas, $\boldsymbol{q}$ es el vector de la onda incidente, $e_{\alpha}$ y $e_{\beta}$ son vectores unitarios en las direcciones cartesianas y $\boldsymbol{k}$ es el momento cristalino. Para calcular la parte real de la matriz dieléctrica $\varepsilon_{\alpha \beta}^{(1)}$, se utilizó la transformación de Kramers-Krönig

$$
\varepsilon_{\alpha \beta}^{(1)}(\omega)=1+\frac{2}{\pi} \wp \int_{0}^{\infty} d \omega^{\prime} \frac{\varepsilon_{\alpha \beta}^{(2)}\left(\omega^{\prime}\right) \omega^{\prime}}{\omega^{\prime 2}-\omega^{2}}
$$

donde $\wp$ denota el valor principal.

\section{RESULTADOS Y DISCUSIÓN}

\subsection{Estructura Cristalina}

La fase cristalina del óxido de niobio $\mathrm{B}-\mathrm{Nb}_{2} \mathrm{O}_{5}$, existe en el rango de presiones entre 5 y $6 \mathrm{GPa}$ y de temperaturas entre 900 y $1000{ }^{\circ} \mathrm{C}$, aproximadamente (Laves et al., 1964; Wadsley \& Anderson, 1970). Esta fase cristaliza en una estructura monoclínica perteneciente al grupo espacial $C_{2} / c$. En la Fig. 1 ilustramos la estructura local de los átomos de oxígeno alrededor de niobio y la celda convencional de $\mathrm{B}-\mathrm{Nb}_{2} \mathrm{O}_{5}$ usando las proyecciones a lo largo de los ejes cristalográficos $b$ y $c$, respectivamente. En Fig. 1(a) se muestra la estructura local del compuesto, donde en el centro se 
encuentra un átomo de $\mathrm{Nb}$ y formando aproximadamente un plano alrededor de él se encuentran cuatro átomos de O. Adicionalmente, se puede observar que los otros dos átomos de oxígeno se encuentran en una línea aproximadamente perpendicular al plano definido por los otros cuatro oxígenos, formando de esta manera una estructura local con una geometría de octaedro distorsionado (Emmeneger \& Robinson, 1968). En la proyección a lo largo del eje $b$ (Fig. 1(b)), se observan los cuatro oxígenos alrededor del átomo de $\mathrm{Nb}$, los cuales componen el plano de un octaedro distorsionado. Adicionalmente, cada octaedro constituido por un átomo de $\mathrm{Nb}$ en su centro comparte dos átomos de $\mathrm{O}$ con el octaedro formado por el siguiente $\mathrm{Nb}$, formando asimismo el arreglo periódico de esta estructura cristalina. En la proyección a lo largo del eje $c$ (Fig. $1(c)$ ), se observa en el centro de la celda convencional un espacio intersticial de considerable extensión que puede ser utilizado en el transporte de iones pequeños, por ejemplo iones de $\mathrm{Li}^{+}$, en esta dirección.

(a)

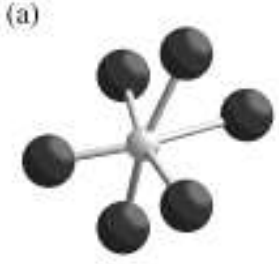

(b)

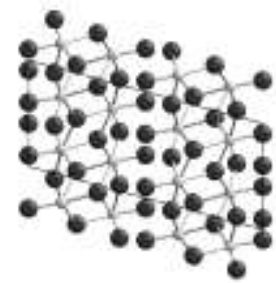

(c)

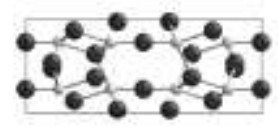

Fig. 1. Celda convencional de $\mathrm{B}-\mathrm{Nb}_{2} \mathrm{O}_{5}$. (a) ilustra la estructura local de los átomos de oxígeno (esferas negras) alrededor de niobio (esferas grises), (b) proyección a lo largo del eje $b$, (c) proyección a lo largo del eje $c$. Fuente: Autores

Los valores teóricos y experimentales de los parámetros cristalográficos (constantes de red, posiciones de Wyckoff y distancias inter-iónicas entre $\mathrm{Nb}$ y $\mathrm{O}$ ) se muestran en las Tablas 1 y 2 , donde se observa que los valores teóricos están en muy buena concordancia con los valores experimentales, mostrando un error relativo de aproximadamente el $2 \%$ para dichos parámetros. Estos parámetros están sobre estimados, como es de esperarse cuando se utiliza la aproximación PBEsol (Perdew et al., 2008). 
Tabla 1. Parámetros cristalográficos experimentales (Laves et al., 1964; Wadsley \& Anderson, 1970) y calculados para B- $\mathrm{Nb}_{2} \mathrm{O}_{5}$ (grupo espacial $C_{2} / c$ ). Fuente: Autores

\begin{tabular}{ccc}
\hline & Experimental & Calculado \\
\hline$a(\AA)$ & 12,43 & 12,829 \\
$b(\AA)$ & 4,88 & 4,8940 \\
$c(\AA)$ & 5,56 & 5,5489 \\
$\beta$ & 105,1 & 104,78 \\
$\Omega_{0}$ & 166,74 & 168,79 \\
$d_{\mathrm{Nb}-\mathrm{O} 2}$ & 1,81 & 1,8143 \\
$d_{\mathrm{Nb}-01}$ & 1,91 & 1,9150 \\
$d_{\mathrm{Nb}-\mathrm{O} 3}$ & 1,94 & 1,9560 \\
$d_{\mathrm{Nb}-02}$ & 2,06 & 2,0458 \\
$d_{\mathrm{Nb}-\mathrm{O} 3}$ & 2,12 & 2,1281 \\
$d_{\mathrm{Nb}-03}$ & 2,19 & 2,1913 \\
\hline
\end{tabular}

Tabla 2. Posiciones de Wyckoff experimentales (Laves et al., 1964; Wadsley \& Anderson, 1970) y calculadas para B- $\mathrm{Nb}_{2} \mathrm{O}_{5}$ (grupo espacial $C_{2} / c$ ). Fuente: Autores

\begin{tabular}{ccccccc}
\hline & \multicolumn{3}{c}{ Experimental } & \multicolumn{3}{c}{ Calculado } \\
\hline & $x$ & $y$ & $z$ & $x$ & $y$ & $z$ \\
$\mathrm{Nb}(8 \mathrm{f})$ & 0,6183 & 0,8991 & 0,2512 & 0,6186 & 0,8996 & 0,2445 \\
$\mathrm{O}_{1}(4 \mathrm{c})$ & 0,8965 & 0,8965 & 0,2488 & 0,8951 & 0,8951 & 0,2500 \\
$\mathrm{O}_{2}(8 \mathrm{f})$ & 0,5509 & 0,3333 & 0,4709 & 0,5587 & 0,3370 & 0,4756 \\
$\mathrm{O}_{3}(8 \mathrm{f})$ & 0,2278 & 0,8668 & 0,1256 & 0,2770 & 0,8658 & 0,1279 \\
\hline
\end{tabular}

\subsection{Propiedades Electrónicas}

En el estudio de la estructura electrónica de $\mathrm{B}-\mathrm{Nb}_{2} \mathrm{O}_{5}$ se ha calculado la densidad de estados (DOS) total y parciales. La Fig. 2 (a) muestra la DOS total del $\mathrm{Nb}_{2} \mathrm{O}_{5}$, donde se puede observar tres bandas principales: bandas de valencia BV1, BV2 y la banda de conducción BC. BV1 está en un rango de energía entre -18 y -15,4 $\mathrm{eV}$ con respecto al máximo de la banda de valencia (MBV), BV2 está en un rango de energía entre -5,3 y $0 \mathrm{eV}$, y BC está localizada a partir de los 2,54 eV y energías superiores; por tanto el gap de energía de este semiconductor es de $2.54 \mathrm{eV}$. En la literatura se reportan dos valores experimentales para el gap de $\mathrm{Nb}_{2} \mathrm{O}_{5}: 3,9 \mathrm{eV}$ 
para la fase amorfa (Madelung et al., 1978) y 3,4 eV para la fase $\mathrm{T}$ (Wachs et al., 2003); estos valores no son directamente comparables con el valor del gap de la fase B, sin embargo, es claro que $\mathrm{Nb}_{2} \mathrm{O}_{5}$ es un semiconductor de gap ancho. Se espera que el valor del gap calculado para $\mathrm{B}-\mathrm{Nb}_{2} \mathrm{O}_{5}$ este subestimado debido a que PBEsol es una aproximación la cual funciona correctamente para el estado base y de una forma aproximada para los estados excitados. La Fig. 2 (b) muestra la DOS parcial de Nb, donde se observa que los estados $d$ son los de mayor contribución y los estados $s$ y $p$ son de menor intensidad con respecto a los estados $d$. La mayor contribución de $\mathrm{Nb}$ está en BV2 y en la BC, la cual está formada en gran parte por los estados $d$ de Nb. La Fig. 2 (c), (d) y (e), muestran las DOS parciales proyectadas sobre los tres oxígenos clasificados por su simetría puntual $\left(\mathrm{O}_{1}, \mathrm{O}_{2}, \mathrm{O}_{3}\right)$. Aquí se observa que la mayor contribución a la DOS está dada por los estados $s$ y $p$, con los estados $s$ formando la BV1 y los estados $p$ se hibridan en BV2 con los estados $d$ de $\mathrm{Nb}$ para formar esta banda. Finalmente, se observa en la DOS parciales que el MBV está formado en su mayor parte por los orbitales $p$ del $\mathrm{O}$, mientras el mínimo de la banda de conducción (MBC) está formado mayoritariamente por los orbitales $d$ de $\mathrm{Nb}$.

Por otra parte, se consideró el espectro de emisión noresonante de rayos-X (XES) el cual da una medida de la contribución de la DOS parciales. En particular, la BV $\mathrm{M}_{4,5}$ (transición $5 p$ $\rightarrow 3 d$ ) de $\mathrm{Nb}$ formada por sus orbitales $5 p$ y la BV Ka (transición $2 p \rightarrow 1 s$ ) de $\mathrm{O}$ formada por sus orbitales $2 p$, pueden ser comparadas con los resultados obtenidos en la Fig. 2. Medidas de XES para B- $\mathrm{Nb}_{2} \mathrm{O}_{5}$ (Kurmaev et al., 2002) son comparadas con las DOS parciales calculadas en las Fig. 3 y 4 , donde primero se ha hecho una convolución de las DOS parciales de acuerdo a la resolución experimental para los estados $5 p$ de $\mathrm{Nb}$ y $2 p$ de $\mathrm{O}$, con una Gaussiana cuya mitad de ancho máximo es de 0,2 y $0,4 \mathrm{eV}$, respectivamente. Se puede observar en la Fig. 3 que la concordancia entre la BV2 y el espectro experimental y la DOS parcial calculada de los estados $5 p$ del $\mathrm{Nb}$ es muy bueno en el ancho de banda y la posición del pico principal experimental localizado a $-1,7 \mathrm{eV}$. Finalmente, la Fig. 4 muestra la concordancia entre el espectro experimental y la DOS parcial calculada de los estados $2 p$ del O en BV2. Se puede 
así concluir que DFT-PBEsol da una descripción adecuada de la banda de valencia cercana a la energía de Fermi de este óxido.

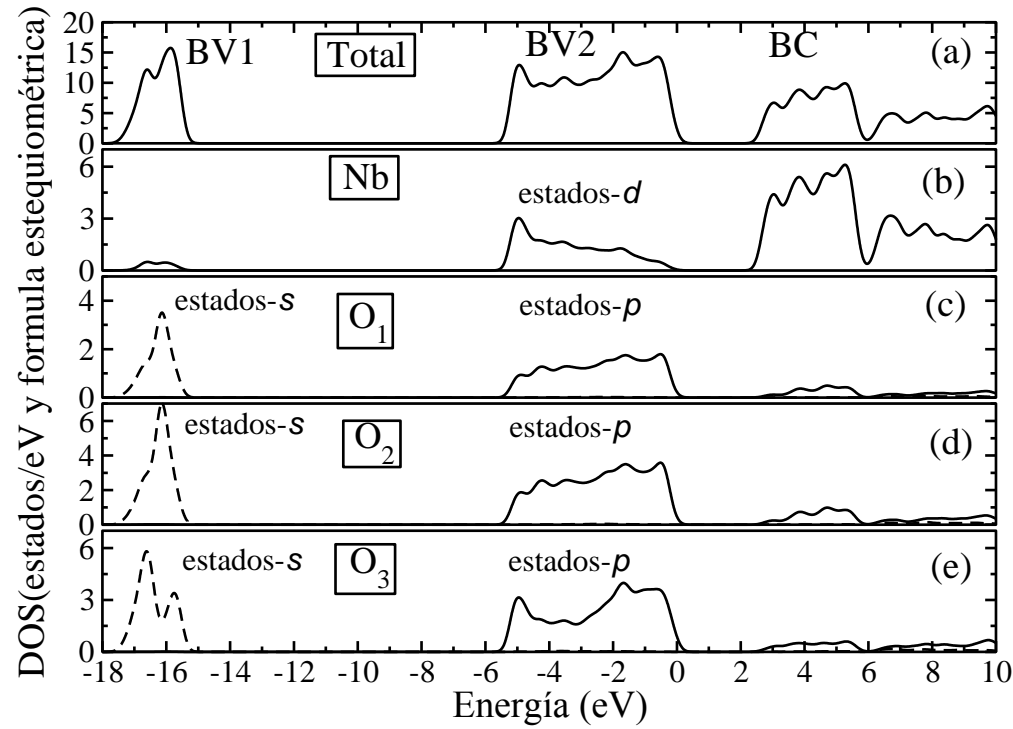

Fig. 2. Densidad de estados (DOS) calculada para B- $\mathrm{Nb}_{2} \mathrm{O}_{5}$. (a) DOS Total, (b) DOS parciales proyectadas sobre $\mathrm{Nb}$, (c)-(e) DOS parciales proyectadas sobre $\mathrm{O}_{1}, \mathrm{O}_{2}$ y $\mathrm{O}_{3}$, respectivamente. Las líneas punteadas en (c)-(e) corresponden a los estados $s$ del $\mathrm{O}$ y las líneas sólidas corresponden a los estados $p$ del O. La energía esta desplazada al máximo de la banda de valencia. Fuente: Autores

\subsection{Propiedades Ópticas}

Las propiedades ópticas de $\mathrm{B}-\mathrm{Nb}_{2} \mathrm{O}_{5}$ fueron determinadas a partir del cálculo del tensor dieléctrico en la celda primitiva, donde sus nueve componentes son reducidas a cuatro componentes debido a la simetría monoclínica del cristal. Estas componentes están a lo largo de los tres ejes cristalográficos (componentes diagonales $\varepsilon_{a}, \varepsilon_{b}$ y $\varepsilon_{c}$ ) y una componente cruzada $\varepsilon_{a c}$, que en este caso es despreciable con respecto a las componentes diagonales. La parte imaginaria de las componentes diagonales $\varepsilon_{a}, \varepsilon_{b}$ y $\varepsilon_{c}$ se muestran en la Fig. 5(a)-(c), respectivamente, donde se puede observar dos picos principales en las tres componentes del tensor dieléctrico 
aproximadamente localizados en $4,3 \mathrm{eV}$ y $8,3 \mathrm{eV}$ para la componente $\varepsilon_{a}, 4,4 \mathrm{eV}$ y $9 \mathrm{eV}$ para la componente $\varepsilon_{b}$ y de $4,4 \mathrm{eV}$ y $8,8 \mathrm{eV}$ para $\varepsilon_{c}$. Además en la Fig. 5 (a)-(c), se observa que la forma de la función dieléctrica es diferente para los tres ejes cristalográficos $\boldsymbol{a}$, $\boldsymbol{b}, \boldsymbol{c}$, revelando el carácter anisotrópico de éste.

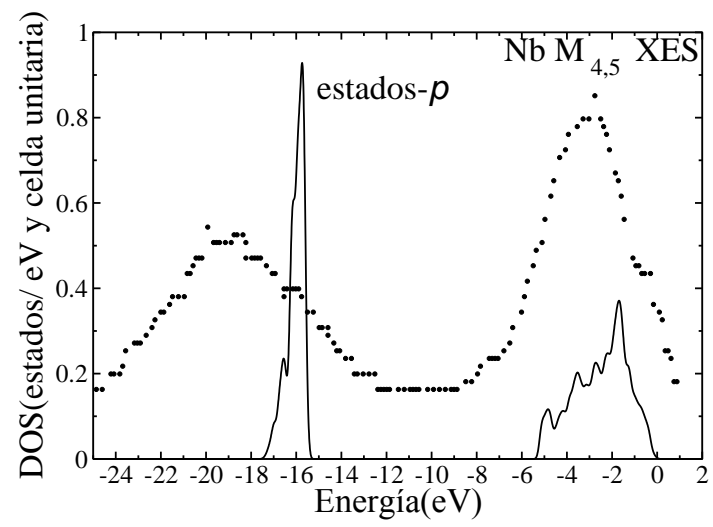

Fig. 3. Comparación entre DOS parcial (estados $p$ ) de Nb y espectro de emisión noresonante de rayos-X (XES) de $\mathrm{B}-\mathrm{Nb}_{2} \mathrm{O}_{5}$. La curva sólida son los valores calculados $\mathrm{y}$ los puntos son los datos experimentales para la $\mathrm{BV} \mathrm{M}_{4,5}$.

Fuente: Kurmaev et al., 2002

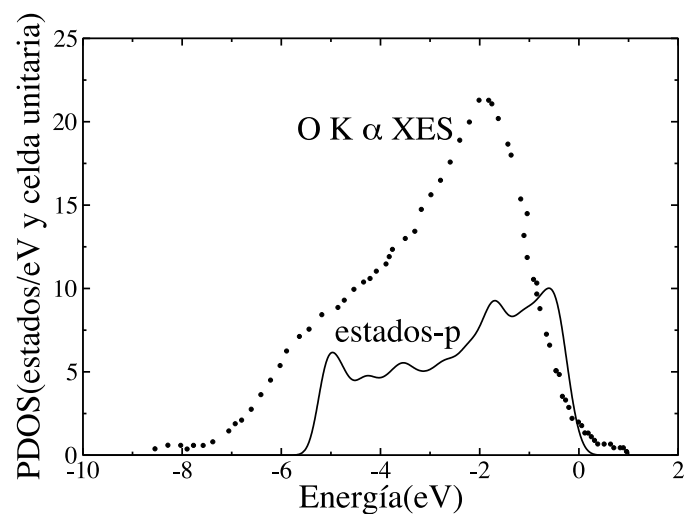

Fig. 4. Comparación entre DOS parcial (estados $p$ ) de $\mathrm{O}$ y XES de $\mathrm{B}-\mathrm{Nb}_{2} \mathrm{O}_{5}$. La curva sólida son los valores calculados y los puntos son los datos experimentales para la BV Ka. Fuente: Kurmaev et al., 2002 
Al comparar los dos picos de máxima intensidad en la Fig. 5 y la DOS total y parciales (Fig. 2), se puede asociar estos dos picos a las transiciones interbandas entre los estados $p$ del $\mathrm{O}$ en la banda de valencia a estados $d$ del $\mathrm{Nb}$ en la banda de conducción. El pico de absorción promedio de las tres componentes del tensor localizado aproximadamente a $4,4 \mathrm{eV}$ (Fig. 5), corresponde aproximadamente a la transición entre los picos localizados a $-1,7 \mathrm{eV}$ localizado en BV2 y 3,0 eV en BC (parte a de la Fig. 2) y el pico de absorción promedio de las tres componentes del tensor localizado aproximadamente a $8,7 \mathrm{eV}$ (Fig. 5), corresponde a la transición entre los picos localizados a $-4,9 \mathrm{eV}$ en $\mathrm{BV} 2$ y $3,8 \mathrm{eV}$ en BC (parte a de la Fig. 2).

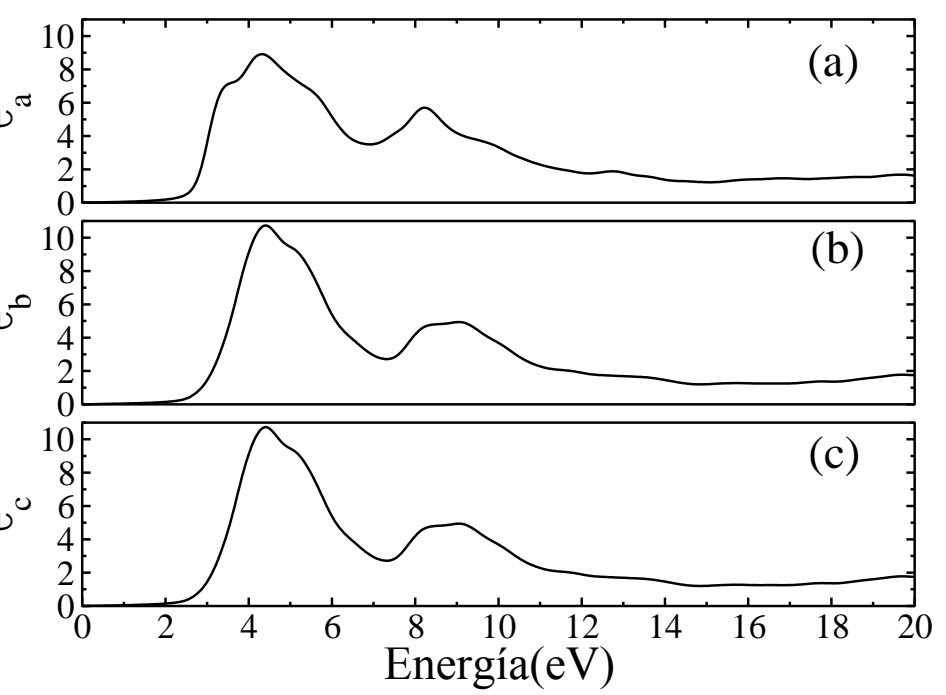

Fig. 5. Parte imaginaria del tensor dieléctrico de $\mathrm{B}-\mathrm{Nb}_{2} \mathrm{O}_{5}$ a lo largo de los tres vectores de Bravais (componentes diagonales $\varepsilon_{a}, \varepsilon_{b}$ y $\varepsilon_{c}$ ). Fuente: Autores

A continuación, se calculó el promedio del índice de refracción $(\tilde{n}=n+i \kappa)$ de las tres direcciones principales. En la Fig. 6(a)-(b), se muestran la parte real $n$ y parte imaginaria $\kappa$ (coeficiente de extinción) de $\tilde{n}$, respectivamente. Se puede observar en la Fig. 6(a) que el valor de $n$ estático (bajas frecuencias) es 2,52, el cual está 
en buena concordancia con el valor experimental de 2,64 (Emmenger et al., 1968). Adicionalmente, se observan regiones de dispersión anómala $\left(\frac{d n}{d \omega}<0\right)$, la cual ocurre cerca de las regiones de absorción entre $5 \mathrm{eV}$ y $9 \mathrm{eV}$. En la Fig. 6(b), se observa que los máximos de absorción de este compuesto están localizados aproximadamente en 5 y $9 \mathrm{eV}$, los cuales están en la región del ultravioleta cercano.

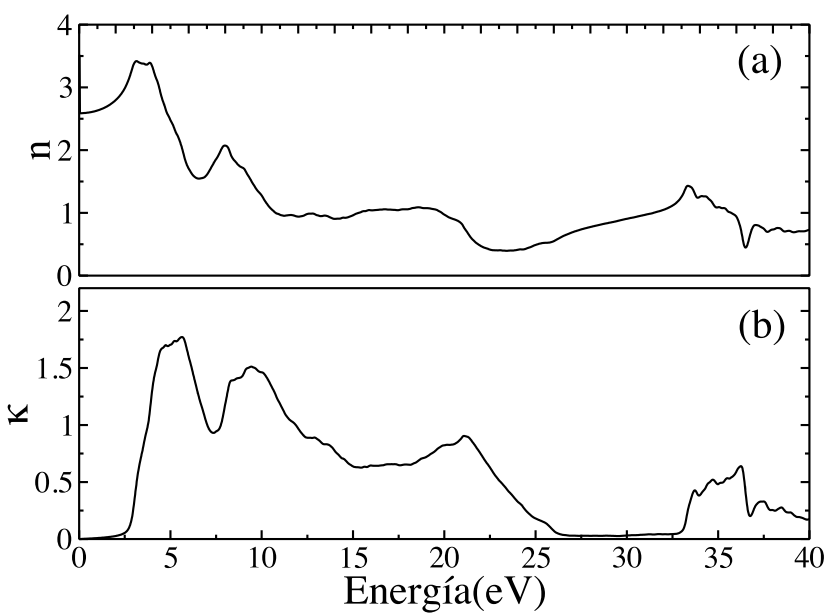

Fig. 6. (a) Parte real y (b) imaginaria del índice de refracción de $\mathrm{B}-\mathrm{Nb}_{2} \mathrm{O}_{5}$. Fuente: Autores

También, se calcularon los coeficientes de reflectividad y transmitancia tal como se muestran en la Fig. 7 (a)-(b), respectivamente, donde se observa que este material es aproximadamente transparente, debido a que en la región del visible entre $0,1 \mu \mathrm{m}$ y 1 $\mu \mathrm{m}$, el coeficiente de transmisión es de $75 \%$ y el coeficiente de reflectividad es de $25 \%$.

Finalmente, se calculó la conductividad óptica real (Fig. 8), donde podemos observar los máximos de absorción localizados en 5 y $9 \mathrm{eV}$, los cuales se deben a la absorción de fotones y de fonones ya que el gap de este sistema es indirecto. 


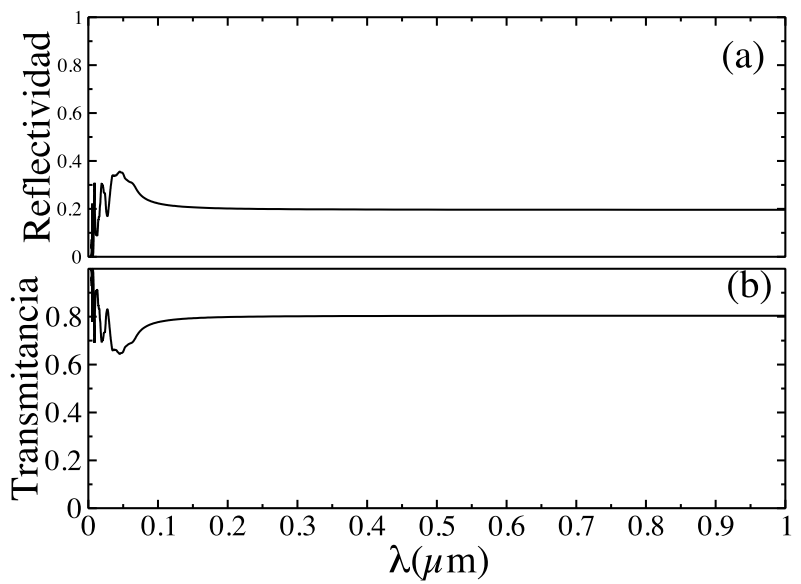

Fig. 7. (a) Reflectividad y (b) Transmitancia de $\mathrm{B}-\mathrm{Nb}_{2} \mathrm{O}_{5}$. Fuente: Autores

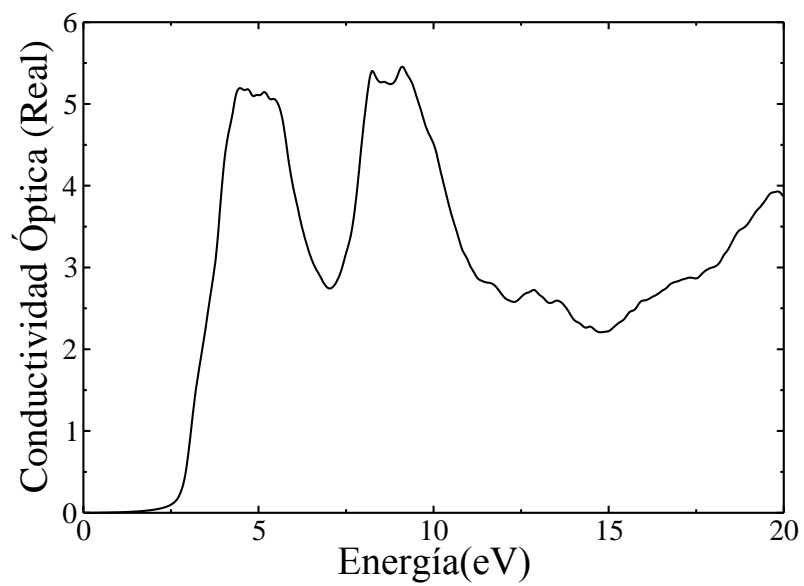

Fig. 8. Parte real de la conductividad óptica de $\mathrm{B}-\mathrm{Nb}_{2} \mathrm{O}_{5}$. Fuente: Autores

\section{CONCLUSIONES}

En este trabajo se estudiaron las propiedades cristalográficas, electrónicas y ópticas del óxido de niobio $\mathrm{Nb}_{2} \mathrm{O}_{5}$ en su fase B. Los resultados teóricos se compararon con los datos experimentales 
reportados en la literatura, donde se observa la buena concordancia entre los datos cristalográficos, mostrando de esta forma que la Teoría de los Funcionales de la Densidad en la Aproximación del Gradiente Generalizado con su parametrización PBEsol, es una excelente herramienta para el estudio de las propiedades cristalográficas de los óxidos de niobio. Adicionalmente, se observó que las características principales de la BV están en buena concordancia cuando comparamos los resultados de XES y los cálculos de DOS parciales. Finalmente, se reportó las propiedades ópticas de este compuesto, y se observó que este formalismo es capaz de predecir de una manera muy acertada el índice de refracción estático de óxido de niobio

\section{REFERENCIAS}

Birch, F., (1947); Finite elastic strain of cubic crystals; Physical Review, $71,809-824$.

Blöchl, P. E., (1994); Projector augmented-wave method, Physical Review B, 50(24), 17953-17974.

Clima, S., y otros 8 autores, (2010); Dielectric response of $\mathrm{Ta}_{2} \mathrm{O}_{5}, \mathrm{Nb}_{2} \mathrm{O}_{5}$, and $\mathrm{NbTaO}_{5}$ from first-principles investigations, Journal of Electrochemical Society, 157(1), G20-G25.

Emmenegger, F. P., \& Robinson, M. L. A., (1968); Preparation and dielectric properties of niobium pentoxide crystals, Journal of Physics and Chemestry of Solids, 29, 1673-1681.

Esteves, A., Oliveira, L. C. A., Ramalho, T. C., Goncalves, M., Anastacio, A. S., \& Carvalho, H. W. P. (2008); New materials based on modified synthetic $\mathrm{Nb}_{2} \mathrm{O}_{5}$ as photocatalyst for oxidation of organic contaminants, Catalysis Communications, 10, 330-332.

Gajdos, M., Hummer, K., Kresse, G., Furthmuller, \& J., Bechstedt, F., (2006); Linear optical properties in the projector-augmented wave methodology, Physical Review B, 73, 045112(1-9).

Kato, V. K., \& Tamura, S., (1975); Die kristallstruktur von T-Nb${ }_{2} \mathrm{O}_{5}$, Acta Crystallographics B, 31, 673-677.

Kresse, G., \& Joubert, D., (1999); From ultrasoft pseudopotentials to the projector augmented-wave method, Physical Review B, 59, 1758-1775. 
Kurmaev, E. Z., Bureev, O. G., Cherkashenko, V. M., Korotin, M. A., \& Ederer, D. L., (2002); Electronic structure of niobium oxides, Journal of alloys and compounds, 347, 213-218.

Laves, F., Petter, W., \& Wulf, H., (1964); Die kristallsturktur von $\mathrm{Nb}_{2} \mathrm{O}_{5}$, Naturwiss, 51, 633-634.

Madelung O., Rössler U., Schulz M. (ed.), (1978); $\mathrm{Nb}_{2} \mathrm{O}_{5}$ : energy gap, optical properties. Collaboration: Authors and editors of the volumes III/17G-41D:. SpringerMaterials - The Landolt-Börnstein Database.

Murnaghan, F. D., (1944); The Compressibility of media under extreme pressures, Proceeding of the National Academy of Sciences, 30, 244247.

Perdew, J. P y otros 7 autores, (2008); Restoring the Density-Gradient Expansion for Exchange in Solids and Surfaces, Physical Review Letter, 100, 136406(1-4).

Prado, A. G. S., Bolzon, L. B., Pedroso, C. P., Moura, A. O., \& Costa, L. L. (2008); $\mathrm{Nb}_{2} \mathrm{O}_{5}$ as efficient and recyclable photocatalyst for indigo carmine degradation, Applied Catalysis B, 82, 219-224.

Santana, V.S., \& Machado, N. R. C. F. (2008); Photocatalytic degradation of the vinasse under solar radiation, Catalysis Today, 133, 606-610.

Sayama, K., Sugihara, H., \& Arakawa, H., (1998); Photoelectrochemical properties of a porous $\mathrm{Nb}_{2} \mathrm{O}_{5}$ electrode sensitized by a ruthenium dye, Chemistry of Materials, 10, 3825-3832.

Tanabe, K., \& Okazaki, S., (1995); Various reactions catalyzed by niobium compounds and materials, Applied Catalysis A, 133, 191-218.

Tanabe, K., (2003); Catalytic application of niobium compounds, Catalysis Today, 78, 65-77.

Wachs, Y. Chen, J. M. Jehng, L. E. Briand, T. Tanaka, (2001); Molecular structure and reactivity of the group V metal oxides, Catalysis today, $78,13-24$.

Wadsley, A. D., \& Andersson, S., (1970); Perspectives in structural chemistry edit. by Dunitz, J. D., and Ibers, J. A., 3, 1, Wiley, New York.

Zibrov, I. P., Filonenko, V. P., Werner, P. E., Marinder, B. O., \& Sundberg, M., (1998); A new high-pressure modification of $\mathrm{Nb}_{2} \mathrm{O}_{5}$, Journal of Solid State Chemestry, 141, 205-211. 
